\title{
Unified low-parametrical equation of state for engineering calculations of thermodynamic properties of substances
}

\author{
Alexander Kaplun ${ }^{\mathrm{a}}$ and Arkadiy Meshalkin \\ Kutateladze Institute of Thermophysics SB RAS, Russian Academy of Sciences, Siberian Branch, \\ Novosibirsk, Russia
}

\begin{abstract}
The new simple semi empirical equation of state for description of $P-\rho-T$ data of "normal" substances was specified. New equation of state has 10 individual adjustable coefficients and it describes thermal properties of gas, liquid and fluid in the main with the accuracy within the error of experimental data, except of critical region. The caloric properties and the speed of sound of argon, nitrogen and carbon dioxide were calculated with the help of known thermodynamic equations and in general divergences between calculated and tabular caloric data do not exceed the experimental error. New equation can be used for engineering calculations at the deficit of experimental data, especially on the caloric properties of substances.
\end{abstract}

It is believed that with development of computer engineering all (or most) problems related to description of the thermodynamic surface of single-component substances can be solved with the aid of formal multivariable equations containing many tens or even hundreds of empirical coefficients. It is difficult to accept this idea, since these equations have a number of well-known fundamental disadvantages.

In this connection the problem of developing relatively simple physically based unified lowparametrical equations of state still seems quite relevant.

In literature there are many (more than 200) low-parametrical equations of state for the singlecomponent substances. The most detailed analysis of these equations is presented in [1].

In previous publications [2-7] we have derived the semi-empirical equations of state, which slightly yield in accuracy of experimental data description to multivariable equations, but they have some physical basis. At that, they take into account both the known and just determined conditions and restrictions, which should be met by the "true" equation of state (see [2-7]). They include the following:

1. The preferred structure of the equation of state was determined [4]. It is shown that the compressibility factor of a single-component substance should contain a term that is a function of density only.

2. The equations for the second virial coefficient of substances with polar and nonpolar molecules were derived in [5] with the help of the modified stepwise potential of intermolecular interaction suggested by us.

\footnotetext{
${ }^{a}$ Corresponding author: kaplun@itp.nsc.ru
}

This is an Open Access article distributed under the terms of the Creative Commons Attribution License 4.0, which permits unrestricted use, distribution, and reproduction in any medium, provided the original work is properly cited. 


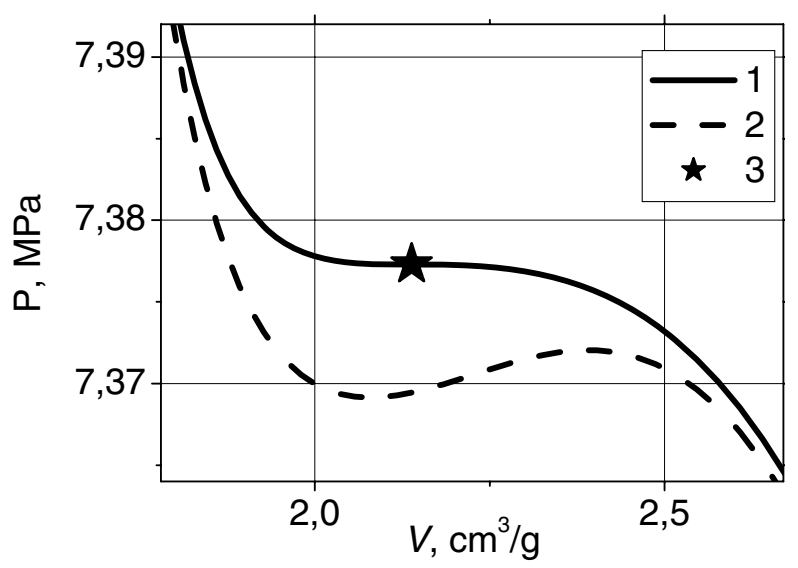

Figure 1. Critical $P-V$ isotherm for carbon dioxide. 1 - calculation by (1); $2-$ calculation by [8]; 3 - critical point.

3. It was determined in [3] with the help of rigorous equations of thermodynamics that isochoric heat capacity (regular part) everywhere on the thermodynamic surface remains finite and positive, including the spinodal.

In the current study a new united equation of state is suggested for liquid, gas and fluid as the development of presented approach for calculation of thermal and caloric properties of substances; this equation describes the experimental data, mainly, within the experimental error. This equation takes form:

$$
\begin{aligned}
Z= & 1+a_{1}\left(e^{a_{2} \cdot \tau}-1-a_{2} \cdot \tau\right)\left(\omega_{t r}-\omega\right)^{2}\left(4 \omega-\omega_{t r}\right) \omega-a_{3} \omega\left(e^{-\tau}-1\right)- \\
& -a_{4} \omega\left(e^{-3 \cdot \tau}-1\right)-a_{5} \omega \tau-a_{6}\left(e^{6 \cdot \tau}-6 \cdot \tau\right)\left(\omega_{t r}-\omega\right)^{3}\left(3 \omega-\omega_{t r}\right) \omega^{2}+ \\
& +\frac{a_{7} \omega}{1-Z_{c} \omega}+\frac{a_{8} \omega^{2}}{\left(1-Z_{c} \omega\right)^{2}}+\frac{a_{9} \omega^{3}}{\left(1-Z_{c} \omega\right)^{3}}+\frac{a_{10} \omega^{4}}{\left(1-Z_{c} \omega\right)^{4}} .
\end{aligned}
$$

Here $Z=P / \rho R T$ is compressibility factor, $P$ is pressure, $\rho=1 / V$ is density, $V$ is specific volume, $T$ is absolute temperature, $R$ is gas constant of substance, $T_{C}$ is critical temperature, $\rho_{C}$ is density of substance at the critical point, $\rho_{t r}$ is density at the triple point, $\tau=T_{c} / T$ is reduced reverse temperature, $\omega=\rho / \rho_{c}$ is reduced density, $\omega_{t r}=\rho_{t r} / \rho_{c}$ is reduced density at the triple point, $a_{1} \ldots a_{10}$ are adjustment coefficients. There are 10 empirical coefficients in equation (1). Coefficients in (1) are determined by the least-squares method by experimental $P-V-T$ data. When coefficients of the sought equation are determined, the values of density at the given temperature and pressure are calculated from this equation numerically. Three classical critical conditions (see Fig. 1) can be strictly satisfied in (1).

While imposing these conditions, the number of varying empirical coefficients is reduced to 7 , and three coefficients are expressed through the others. We should emphasize that in low-parametrical equations of state the critical conditions (without loss of accuracy at experimental data description) were obtained for the first time.

Deviations of carbon dioxide densities, calculated by (1) from the tabular data of [12] are shown in Fig. 2 as an example. According to Fig. 2, divergence between the values calculated by (1) and tabular data [12] in a wide range of the state parameters do not exceed $0.2 \%-0.3 \%$, excluding data in the near-critical area.

As it is known, the caloric properties of substances, such as enthalpy, entropy, heat capacity, etc. can be calculated by the differential thermodynamic equations for the thermal data using equation of 
Thermophysical Basis of Energy Technologies

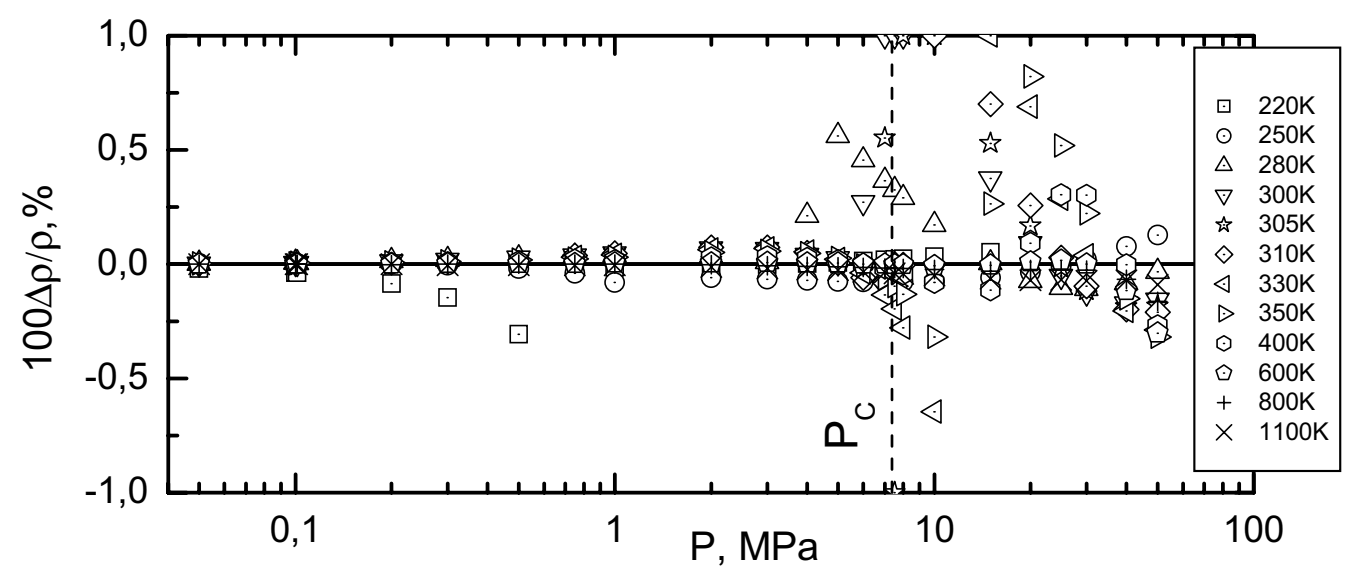

Figure 2. Deviation of carbon oxide densities, calculated by (1), from the tabular data, calculated by the standard EOS [12] (185 adjustable coefficients). Above $50 \mathrm{MPa}$ - extrapolation by (1).

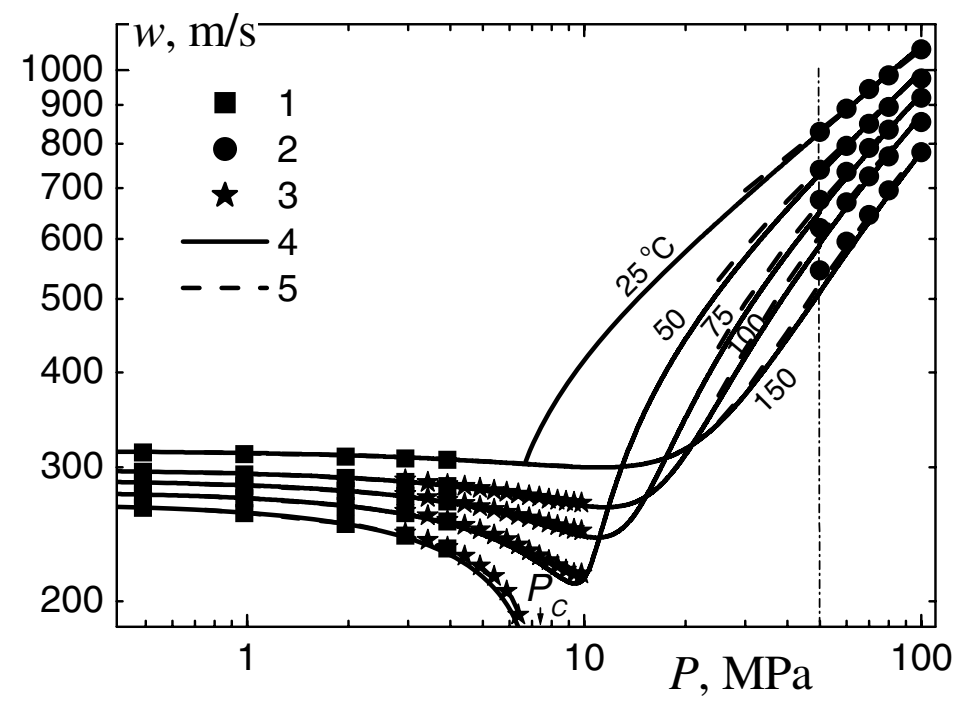

Figure 3. Comparison of sound velocities in $\mathrm{CO}_{2}$ calculated by (1) with experimental data: 1 - data of [10]; 2 - data of [9]; 3 - data of [11]; 4 - calculation by (1) (10 coefficients); 5 - calculation by [12] (185 coefficients). From 50 to $100 \mathrm{MPa}$ - extrapolation by (1).

state (1). We cannot give here the detailed analysis of results of this calculation because of the limited space. We will be confined by the results of a-priori calculation of the equilibrium velocity of sound in $\mathrm{CO} 2$ shown in Fig. 3 as an illustration. We should emphasize that no any data on sound velocity and caloricity were used for this calculation, excluding the enthalpy of ideal gas.

\section{References}

[1] G.A. Spiridonov, I.S. Kvasov, Reviews of thermal-physical properties of substances. 57, 45 (1986)

[2] A.B. Kaplun, A.B. Meshalkin, J. Chem. Eng. Data, 55, 4285 (2010) 
[3] A.B. Kaplun, A.B. Meshalkin, High Temperature, 46, 383 (2008)

[4] A.B. Kaplun, A.B. Meshalkin, High Temperature, 41, 373 (2003)

[5] A.B. Kaplun, A.B. Meshalkin, Russ. J. Phys. Chem., 80, 2097 (2006)

[6] A.B. Kaplun, A.B. Meshalkin, J. Chem. Eng. Data, 56, 1463 (2011)

[7] A.B. Kaplun, A.B. Meshalkin, High Temperature, 48, 692 (2010)

[8] R. Span, R. Wagner, Intern. J. Thermophysics, 24, 1 (2003)

[9] I.I. Novikov, Yu.S. Trelin, Thermal Engineering, 9, 78 (1962)

[10] V.A. Gruzdev, V.I. Slabnyak, Investigation of thermal-physical properties of substances. Collected papers. (Nauka, Novosibirsk, 1970)

[11] L.L. Pitaevskaya, A.V. Bilevich, Russ. J. Phys. Chem., 47, 227 (1973)

[12] R. Span, R. Wagner, J. Phys. Chem. Ref. Data, 25, 1509 (1996) 